\title{
A Generic And Configurable Topology Discovery Service For Software Defined Wireless Multi-Hop Network
}

\author{
Lunde Chen \\ Slim Abdellatif \\ CNRS, LAAS, 7 avenue du colonel \\ Roche, F-31400 Toulouse, France \\ Univ de Toulouse, INSA, LAAS, \\ F-31400 Toulouse, France
}

\author{
Pascal Berthou \\ Kokouvi Bénoît Nougnanke \\ CNRS, LAAS, 7 avenue du colonel \\ Roche, F-31400 Toulouse, France \\ Univ de Toulouse, UPS, LAAS, \\ F-31400 Toulouse, France
}

\author{
Thierry Gayraud \\ CNRS, LAAS, 7 avenue du colonel \\ Roche, F-31400 Toulouse, France \\ Univ de Toulouse, UPS, LAAS, \\ F-31400 Toulouse, France
}

\begin{abstract}
We present a topology discovery service for software-defined wireless multi-hop networks, which is capable of capturing rich information about the network topology, such as link quality, interference and node characteristics etc., to effectively support the requirements of higher-layer SDN applications such as routing, channel allocation and transmission power control with enhanced flexibility. We analyse the general topology representation required by such SDN applications and specify the procedure and mechanism required at the controller and nodes to maintain this representation. A proof-of-concept implementation of our service on an SDN enabled multi-channel multi-interface wireless multi-hop network testbed shows the feasibility of our proposal.
\end{abstract}

\section{KEYWORDS}

wireless SDN, link quality metric, topology discovery service, OFDP, Open vSwitch

\section{INTRODUCTION}

Software-Defined Networking (SDN) has recently emerged as a new paradigm with the idea of taking control plane functions out of network forwarding devices and relocating them on remote external computer machines. The SDN architecture introduces a new entity, called the controller, that interfaces between the network control functions (or network control applications) and the network devices. It typically exposes, on the one hand, network or topology discovery services which provide these latter applications with the appropriate view of the network, and, on the other hand, some interfaces that let network control applications program remotely the forwarding devices.

Network and topology discovery services are essential to SDN applications. They require that the controller maintains a comprehensive view of the network (a network-wide view of forwarding devices, their intrinsic properties, and their status) from which it can derive different views, cutsomized to each control application. The OpenFlow protocol, the main SDN enabler, doesn't specify how to proceed with the topology discovery. Current SDN controllers such as Ryu[1], OpenDaylight[2] etc. implement OFDP (OpenFlow Discovery Protocol)[3], which leverage LLDP (Layer Discovery Protocol)[4] with subtle modifications to perform topology discovery in an OpenFlow network. Since SDN applications were centred on wired networks, to the best of our knowledge, the network representation that current SDN controllers typically build is limited to the network topology with additional informations related to forwarding nodes and their network interfaces (MAC and IP level addresses, speed, etc.). This is clearly not sufficient in a wireless SDN context. For instance, such a network representation assumes that links either work well or do not work at all and, hence, fails in capturing crucial characteristics of wireless links, namely the intermediate and varying performance of wireless links (as opposed to the $0-1$ binary representation of connectivity between wireless nodes).

In this paper, we propose a new topology discovery scheme that is able to support effectively the various needs of the network control applications that may be used in a multi-hop wireless SDN context. The proposed scheme allows the controller to keep an updated, detailed and adaptive knowledge of the network. This network view includes: the connectivity between nodes, the states and performance of links and nodes, and the intrinsic properties of nodes and links. This work also presents how this scheme can be implemented on SDN controllers and switches.

This paper is organised as follows. Section 2 presents existing works related to the topology discovery in a wired and wireless SDN context. Sections 3 and 4 respectively present the proposed scheme and a prototype set-up with some experimental results. Section 5 concludes the paper.

\section{RELATED WORK}

The state-of-art SDN controllers use OFDP, which leverages the Link Layer Discovery Protocol (LLDP) with subtle modifications to perform link discovery in an OpenFlow network. The controller sends periodically a separate Packet-Out message with a dedicated LLDP packet for each port of each switch to discover their one-hop neighbours, allowing it to discover all available links in the network. Adapting OFDP directly for a wireless SDN context, however, has several limitations that we will detail in the next section.

State-of-art work on multi-hop wireless SDN discovers the topology by exchanging broadcast packets between switches to learn their one-hop neighbours, then upload such information to the SDN controller [5] [6] [7]. Most implicitly assume a 0-1 binary representation of wireless links. Other wireless SDN solutions [8] [9] propose gathering information such as RSSI, battery level etc, but much knowledge about the network is still missing, especially those regarding to interference and channel usage. 


\section{THE PROPOSED DISCOVERY SCHEME}

\subsection{Network representation at the controller}

Many network control functions will be using the network/topology discovery service, notably routing, channel assignment and topology control. We briefly and broadly describe the main requirements that has led to the network representation that we propose.

3.1.1 QoS routing. In a wireless multi-hop network, one of the most important and challenging functionality is routing. To guide the selection of routes, link metrics should be used and chosen according to application requirements, network characteristics (mono or multi-interface, node mobility, energy constrained nodes, etc.) and the environment in which the network operates. As a consequence, our network representation supports multiple routing metrics by adaptively collecting a set of link and node information that can be combined to derive state-of-the-art wireless link metrics. When possible, it also includes some node attributes related to the available energy or the GPS position which is exploited by geographic routing algorithms.

3.1.2 Channel assignment. The problem of channel assignment is to assign a unique channel to each communication link in the network such that the interference is minimized. Channel assignment algorithms usually rely on conflict graphs to assign channels to wireless nodes. The channel (or frequency band) on which each network interface operates should be made available and hence included in the network representation. The diverse wireless links metrics should also be used to assess the level interference of each link.

3.1.3 Transmission power control. Topology control is the adjustment of node parameters and modes of operation so as to modify the topology of the network [11]. Transmission power control requires usually the knowledge of the transmission power of each node in the network, and the connectivity between them and the associated link channel/frequency. Also, the locations of wireless nodes may be needed as in [12], as well as the received SINR [13].

3.1.4 Summary of our proposed topology representation. The requirements of the three presented network applications are quite representative of what network control applications may expect from a network discovery service. These are expressed as nodes and link attributes to be collected by the SDN controller's topology discovery manager regarding to their quality, channel usage, localization and transmission power etc.

\subsection{Topology construction and maintenance}

Two design principles are at the heart of our proposed topology discovery service : (1) genericity in the sense that a plenty of nodes and link attributes can be made available to a variety of network control applications; (2) configurability in the sense that the network topology that is built at the controller is configured and set according to the needs of network control applications that are running. From these needs, the network topology discovery service of the controller derives the attributes to collect. It consequently configure application agents running on the wireless nodes to proceed with local configurations as well as some measurements at nodes or wireless links to which nodes belong. The collected values are then sent to the controller following some procedures,

The general behaviour of the proposed topology discovery service relies on and extends the de facto service implemented by many common SDN controllers for wired networks. It mainly proposes some modifications and extensions to make it work effectively for wireless multi hop networks, namely the OpenFlow protocol and the OpenFlow Discovery Protocol (OFDP). The de facto topology service was briefly presented in Section 2, we detail hereafter its main limitations before presenting the proposed extensions. Referring to previous sections, the existing topology discovery service has three main limitations to be applied to wireless multi-hop networks. These limitations are mainly due to the fact that the service has been implicitly designed for wired networks :

(1) the lack of some node related attributes such as localization, remaining energy, etc. but also wireless interface related attributes such as the channel on which the interface operates at a given time, the transmission power, the antenna characteristics, etc.

(2) the assumption that links are point-to-point which do not hold for wireless links

(3) the binary sate $(\mathrm{On} / \mathrm{Off})$ of links which is not sufficient to capture the intermediate and varying performance of wireless links.

Our proposal described hereafter addresses these limitations.

3.2.1 Wireless node and port related attributes integration. The OpenFlow session establishment, which is initiated by the OpenFlow wireless node, goes through a sequence of steps : OpenFlow version negotiation, node and then port features discovery. Most of the missing nodes attributes are dynamic and requires node to controller exchanges to keep these attributes up-to-date. Our proposal is to use the OpenFlow EchoRequest / EchoReply procedure to convey these attributes. These are symmetric messages and can be initiated by a node at will.

Static port attributes are discovered with the "OFPT_MULTIPART _REQUEST/REPLY" procedure which includes a "OFPMP_PORT_DE SC" structure that gathers port characteristics. Since wireless ports are not yet considered by the OpenFlow 1.5.1 specification, we have proposed the attributes depicted on Listing 1 for WiFi interfaces. The static attributes are discovered following the procedure described above while the dynamic ones are maintained using the "OFPT_PORT_STATUS" procedure of the OpenFlow protocol.

3.2.2 Effective wireless link discovery with OFDP. OFDP messages are actually LLDP frames encapsulated in an OpenFlow packetout message, generated by SDN controller. Each LLDP frame starts with the following mandatory TLVs: Chassis ID, Port ID, and Timeto-Live. The mandatory TLVs are followed by optional TLVs. The frame ends with a special TLV named end of LLDPDU.

Links in a wireless network tend to be multipoint links. The transmission of an LLDP message over a wireless port of a given node can be received by several nodes operating on the same channel. As a result, several OpenFlow packet-in messages linked to the same LLDP message can go back to the controller. Each message received by the controller is interpreted as a link change due to 
the implicit assumption of point-to-point wired links. Thus, the controller discovery service notifies a succession of link changes when it should have discovered the presence of several links between each pair of nodes. Indeed, the controller operation is as follows : When a packet-in is received, the controller checks if it is an LLDP frame. If so, it retrieves the information present in this frame, namely source node, destination node and their ports. It then fetches from the list of existing links an entry that matches the source address and its outgoing port. If the link does not exist, a new link is added. If it exists, and the destination address does not coincide, the link is considered as obsolete link (Old_Peer) and replaced with the new one which is incorrect in a wireless context.

In order to make the topology discovery functional for wireless ad-hoc networks, we modify the algorithm in such a way that an old link event (Old_Link_Event) is only triggered if a link considered as existing (present in the All_Links link list) is not updated at the end of an issued LLDP packet number.

3.2.3 Wireless link metrics integration. As explained above, agents are configured and deployed on wireless nodes in order to measure the required wireless link performance metrics. The values collected at the nodes are then delivered to the controller. Our approach is to take advantage of the OFDP packets that are sent periodically for link discovery and maintenance by exploiting the optional TLVs of LLDP packets (one TLV per performance metric). When sending an OFDP message, the controller adds the optional TLVs related to the performance metrics to be updated. When receiving the OFDP message and parsing the optional TLVs, a node deduces the performance metrics that are actually needed by the controller and updates the TLVs with the last measured value and then transmits the OFDP packet back to the controller.

This procedure requires from the wireless nodes to process LLDP messages (parse and modify its fields). In practice, a standard Open vSwitch enabled node do not have this capability since only two operation modes with respect to LLDP messages have been implemented : "LLDP disabled mode" which is the default mode when OpenFlow is enabled, in which LLDP are simply forwarded by the node to the controller without any processing , and the "LLDP enabled mode" which corresponds to the standard use of LLDP where LLDP fields are generated by the switching nodes and processed by the receiving neighbouring node but never forwarded. For our proposed topology discovery service, we have defined a new intermediate operation mode, where switching nodes are not allowed to generate LLDP packets (this is the responsibility of the controller), but are allowed to process and forward LLDP packets.

Listing 1: WiFi port description property

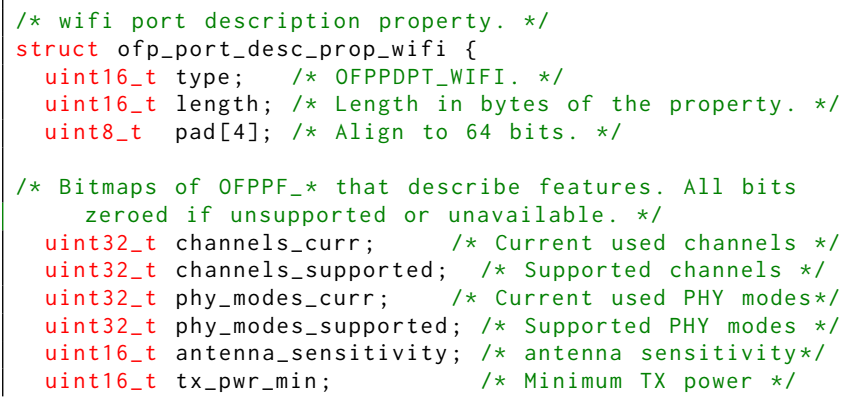

3.2.4 Some opportunities of the proposed service. Our proposition has also the advantage of being flexible and innovation encouraging. This finds its root in the system architecture which has a global view of the network and the flexible combination of collected link and node attributes. One use case is to adaptively select suitable link quality metrics, as different network applications could require different topology information to function. Another use case is that new link quality metrics could be developed with the global view of the SDN controller and the diverse link and node attributes collected.

\section{PROTOTYPE AND EVALUATION}

\subsection{Testbed set-up}

A testbed has been setup to validate this concept in the premises of our lab. It consists in 17 OpenWRT nodes with one to three WiFi interfaces, which can operate at different channels. The OpenFlow support has been added with Open vSwitch bridge installation on each of them. The testbed is configured to connect the nodes to the controller, either directly using a dedicated network (out-of-band control), either using a multi-hop connexion to reach the controller (in-band control), as in shown in Figure 1 and Figure 3. Source codes of Ryu and OVS are modified as is presented in Section 3, to support the modification, addition and parsing of TLVs in LLDP packets.

\subsection{Platform evaluation}

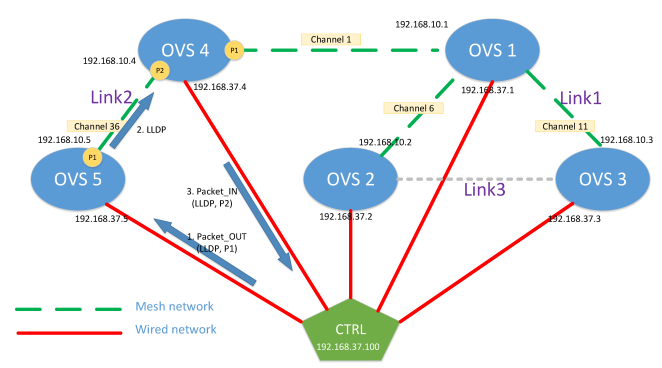

Figure 1: Out-of-band control network

Control traffic between controller and nodes is at the heart of the operation of an SDN network architecture. As a result, most of the traffic exchanged by these communications must be delivered with a certain level of performance, in terms of reliability and delay. We therefore evaluate the the delay of link discovery and the throughput of traffic control messages, the latter could be an important factor of performance when there is interference.

Figure 4 represents the delay of link discovery. It is minimal when these packets are sent with a dedicated out-band traffic control network (less than $10 \mathrm{~ms}$ ), but could increase if the node have to handle also data traffic. The delay is higher if this control traffic is sent in-band. Also, it takes more time to discover 2-hops-way links than one-hop-way links. 
The throughput of OpenFlow control traffic also increases when number of nodes and links increases, as is shown in Figure 2. The volume remains under $10 \mathrm{Kbps}$ for small topologies of less than 12 nodes.

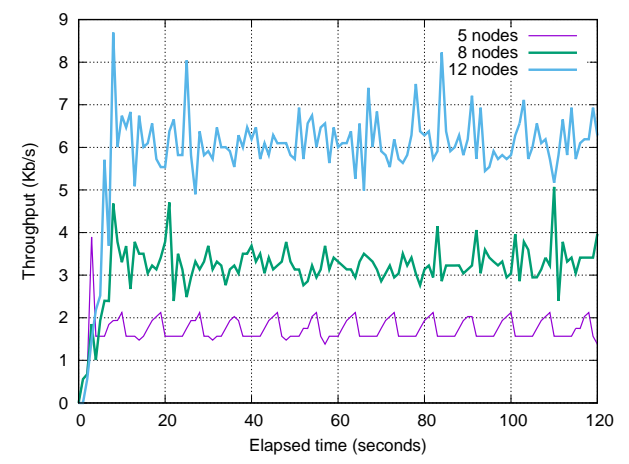

Figure 2: OpenFlow control traffic throughput

\section{CONCLUSIONS}

In this paper, we have proposed a topology discovery service for SDN based wireless multi-hop networks. The proposed service can be used by common network control applications and opens the way to the design of novel algorithms that take benefit from : (1) the centralized view and the rich information exposed by our proposed service, (2) the fine grained programming capabilities of SDN. The proposed service extends and adapts the procedures of the de facto topology discovery service implemented in most SDN/OpenFlow controllers for wired networks, more precisely OFDP/LLDP and the OpenFlow protocol. The service was implemented on the Ryu controller with Open vSwitch enabled wireless nodes.

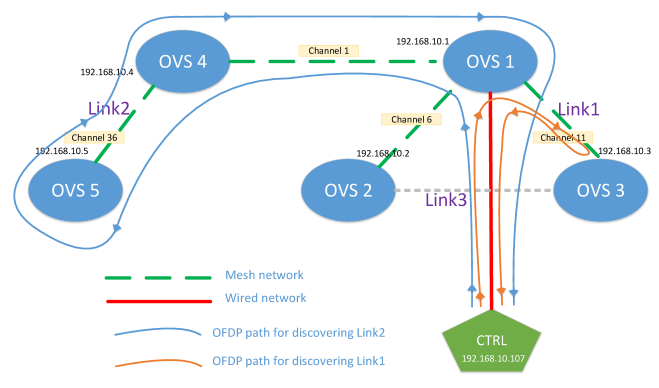

Figure 3: In-band control network

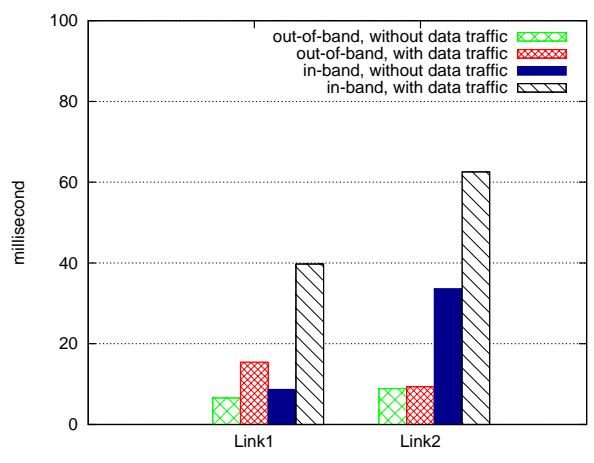

Figure 4: Delay of OFDP messages from packet-out to packetin for discovering two different links

\section{ACKNOWLEDGMENT}

This work was partially funded by the French National Research Agency (ANR) and the French Defense Agency (DGA) under the project ANR DGA ADN (ANR-13-ASTR- 0024) and by European Union's Horizon 2020 research and innovation programme under the ENDEAVOUR project (grant agreement 644960).

\section{REFERENCES}

[1] "Ryu Controller", [Online]. Available: osrg.github.io/ryu

[2] "OpenDaylight Controller", [Online]. Available: www.opendaylight.org

[3] OpenFlow Discovery Protocol and Link Layer Discovery Protocol, [Online]. Available: groups.geni.net/geni/wiki/OpenFlow DiscoveryProtocol

[4] IEEE Station and Media Access Control Connectivity Discovery, IEEE Standard 802.1 AB (LLDP), 2009.

[5] I. Ku, Y. Lu and M. Gerla, "Software-Defined Mobile Cloud: Architecture, services and use cases," in Wireless Communications and Mobile Computing Conference (IWCMC), 2014 International, Nicosia, 2014, pp. 1-6.

[6] P. Dely, A. Kassler and N. Bayer, "OpenFlow for Wireless Mesh Networks," in Computer Communications and Networks (ICCCN), 2011 Proceedings of 20th International Conference on, Maui, HI, 2011, pp. 1-6.

[7] Yuhuai Peng, Lei Guo, Qingxu Deng, Zhaolong Ning and Lingbing Zhang, "A Novel Hybrid Routing Forwarding Algorithm in SDN Enabled Wireless Mesh Networks," in 2015 IEEE 7th International Symposium on High Performance Computing and Communications (HPCC), New York, NY, 2015, pp. 1806-1811.

[8] L. Galluccio, S. Milardo, G. Morabito and S. Palazzo, "SDN-WISE: Design, prototyping and experimentation of a stateful SDN solution for WIreless SEnsor networks," in Computer Communications (INFOCOM), 2015 IEEE Conference on, Kowloon, 2015, pp. 513-521.a

[9] B. T. de Oliveira, C. B. Margi and L. B. Gabriel, "TinySDN: Enabling multiple controllers for software-defined wireless sensor networks," in Communications (LATINCOM), 2014 IEEE Latin-America Conference on, Cartagena de Indias, 2014, pp. 1-6.

[10] R. Fonseca, O. Gnawali, K. Jamieson, and P. Levis. Four Bit Wireless Link Estimation. in Hotnets-VI, Atlanta, GA, Nov. 2007.

[11] Labrador, Miguel A., Wightman, Pedro M. "Topology Control in Wireless Sensor Networks", 2009, Springer

[12] R. Ramanathan and R. Rosales-Hain, "Topology control of multihop wireless networks using transmit power adjustment," in INFOCOM 2000. Nineteenth Annual foint Conference of the IEEE Computer and Communications Societies. Proceedings. IEEE, Tel Aviv, 2000, pp. 404-413 vol.2.

[13] L. P. Qian, Y. J. Zhang and J. Huang, "MAPEL: Achieving global optimality for a non-convex wireless power control problem," in IEEE Transactions on Wireless Communications, vol. 8, no. 3, pp. 1553-1563, March 2009. 